\title{
Chirp Mitigation of Plasma-Accelerated Beams by a Modulated Plasma Density
}

\author{
R. Brinkmann, ${ }^{1}$ N. Delbos, ${ }^{2}$ I. Dornmair, ${ }^{2}$ M. Kirchen, ${ }^{2}$ R. Assmann, ${ }^{1}$ C. Behrens, ${ }^{1}$ K. Floettmann, ${ }^{1}$ J. Grebenyuk, ${ }^{1}$ \\ M. Gross, ${ }^{3}$ S. Jalas, ${ }^{2}$ T. Mehrling, ${ }^{1}$ A. Martinez de la Ossa, ${ }^{4}$ J. Osterhoff, ${ }^{1}$ B. Schmidt, ${ }^{1}$ V. Wacker, ${ }^{1}$ and A. R. Maier ${ }^{2, *}$ \\ ${ }^{1}$ Deutsches Elektronen-Synchrotron DESY, Notkestrasse 85, 22607 Hamburg, Germany \\ ${ }^{2}$ Center for Free-Electron Laser Science and Department of Physics, University of Hamburg, \\ Luruper Chaussee 149, 22761 Hamburg, Germany \\ ${ }^{3}$ Deutsches Elektronen-Synchrotron DESY, Platanenallee 6, 15738 Zeuthen, Germany \\ ${ }^{4}$ Institut für Experimentalphysik, Universität Hamburg, 22761 Hamburg, Germany
}

(Received 8 December 2015; published 23 May 2017)

\begin{abstract}
Plasma-based accelerators offer the possibility to drive future compact light sources and high-energy physics applications. Achieving good beam quality, especially a small beam energy spread, is still one of the major challenges. Here, we propose to use a periodically modulated plasma density to shape the longitudinal fields acting on an electron bunch in the linear wakefield regime. With simulations, we demonstrate an on-average flat accelerating field that maintains a small beam energy spread.
\end{abstract}

DOI: 10.1103/PhysRevLett.118.214801

Using the extreme field gradients supported by a plasma cavity [1], plasma-based accelerators [2] promise very compact sources of ultrarelativistic electron beams for a large variety of applications. Yet, especially the beam energy spread, which in plasma experiments is typically on the percent level [3], causes emittance growth during beam transport, and hence renders its applicability to novel freeelectron laser (FEL) schemes [4,5] or high-energy physics applications [6] very difficult. Controlling the beam energy spread is thus one of the major challenges in the field of plasma acceleration.

Decoupling the generation of the beam from the acceleration allows us to independently control and optimize the dynamics of each process and thus improve the beam quality. Prominent examples of this approach are based on the linear or quasilinear wakefield regime [2], and include the external injection of a well-characterized and tuned electron beam into a plasma acceleration stage, the more general concept of a staged plasma accelerator [6], and the colliding pulse injection scheme [7]. The community has made great progress in the direction of decoupling injection and acceleration with the recent experimental demonstration of a staged plasma accelerator [8] and by discussing the preservation of beam quality coupling the beam into and out of a plasma [9-12].

In a plasma wakefield the beam is typically located at the slope of the accelerating field (referred to as off-crest acceleration), such that it is simultaneously accelerated and focused by the plasma fields. However, this choice

Published by the American Physical Society under the terms of the Creative Commons Attribution 4.0 International license. Further distribution of this work must maintain attribution to the author(s) and the published article's title, journal citation, and DOI. of accelerating phase also imprints a longitudinal energy correlation (chirp) onto the bunch - an intrinsic feature of virtually all plasma-acceleration schemes, and a major source of the undesired energy spread growth.

Here, we propose a novel scheme based on a periodically modulated plasma density profile in the linear wakefield regime, which mitigates the energy chirp accumulation in the plasma. By modulating the plasma density, the bunch periodically experiences accelerating fields with opposite slope, which effectively suppresses the chirp evolution. Our concept of periodically modulating the plasma density allows us to actively shape the longitudinal fields inside the plasma wave with applications even beyond the removal of the linearly correlated energy spread.

This Letter is structured as follows. First, we discuss the longitudinal and transverse fields in the plasma cavity and derive an expression for the beta function that is matched to the focusing of a periodically modulated plasma density. We then demonstrate the chirp mitigation of our scheme using particle-in-cell (PIC) simulations for a laser- and a beam-driven plasma wakefield and compare it to a reference case of constant plasma density.

In the following, we assume a linear plasma wakefield generated by a Gaussian driver described by $f(r, \zeta)=$ $f_{0} \exp \left(-r^{2} / 2 \sigma_{r}^{2}\right) \exp \left(-\zeta^{2} / 2 \sigma_{z}^{2}\right)$. Our discussion applies to both laser-driven wakefields, where $f_{0}=a_{0}^{2} / 4$ for a linearly polarized laser pulse, and particle beam-driven wakefields, with $f_{0}=n_{b} / n_{e}$ for an electron beam. Here, $n_{b}$ is the density of the driver beam, while $n_{e}$ denotes the plasma electron density. The plasma wave number is $k_{p}=\omega_{p} / c$, with $\omega_{p}=\left(n_{e} e^{2} / \epsilon_{0} m_{e}\right)^{1 / 2}$, and $\zeta=z-c t$ the distance behind the driver. The peak normalized laser vector potential is $a_{0}=e A_{0} / m_{e} c^{2}$. In the linear wakefield regime, $a_{0}^{2} \ll 1$ for a laser driver and $n_{b} / n_{e} \ll 1$ for an electron beam driver. In an external injection scheme, the 
electron bunch (witness bunch) may be positioned at an arbitrary phase $\Psi=k_{p} \zeta$ of the plasma wave. The wakefields behind the driver are $[2,13]$

$$
\begin{aligned}
E_{z}(r, \zeta) & =E_{z, 0} \exp \left(-\frac{r^{2}}{2 \sigma_{r}^{2}}\right) \cos \left(k_{p} \zeta\right), \\
E_{r}(r, \zeta)-c B_{\theta}(r, \zeta) & =-E_{z, 0} \frac{r}{k_{p} \sigma_{r}^{2}} \exp \left(-\frac{r^{2}}{2 \sigma_{r}^{2}}\right) \sin \left(k_{p} \zeta\right),
\end{aligned}
$$

with $E_{z, 0}$ the amplitude of the longitudinal field component [2]. The focusing strength $K$ acting on a relativistic electron bunch in the vicinity of the axis, with $\gamma_{L}$ the relativistic Lorentz factor, is

$$
K(\zeta)=\left.\frac{e}{\gamma_{L} m_{e} c^{2}} \partial_{r}\left(E_{r}-c B_{\theta}\right)(r, \zeta)\right|_{r=0} .
$$

It would be desirable to position the witness bunch on crest, i.e., at the maximum of the accelerating field at $\Psi=-\pi$, which avoids, to first order, a gradient of the accelerating field along the bunch. Yet, for plasma accelerators, the bunch tail would erode due to the defocusing fields for $\Psi<-\pi$. For off-crest acceleration, within the interval of $-\pi<\Psi<-\pi / 2$, the transverse fields focus the whole electron beam, while it is accelerated by the longitudinal field $E_{z}(\zeta)$. This allows us to transport the beam through the plasma, albeit at the cost of a correlated energy spread, caused by the gradient in $E_{z}$.

The effect can be mitigated with smaller ratios of $\sigma_{z} / \lambda_{p}$, i.e., using shorter bunch lengths $\sigma_{z}$, or lower plasma densities, i.e., longer $\lambda_{p}$. Yet, ever shorter electron bunches are difficult to generate in a conventional rf-based accelerator, and also they support much smaller beam charge. In addition, lower plasma densities require significantly higher laser pulse energies to resonantly drive the wake, limit the bunch density as beam loading effects become more severe, and decrease the accelerating gradient. An interesting concept proposes a hollow plasma channel free of transverse focusing forces to position a witness bunch on crest [14]. Alternatively, it has been proposed to utilize the beam loading field of the electron beam in the plasma to flatten $E_{z}$ along the bunch [15]. However, this requires very precise control over the injected current profile to exactly cancel the gradient in $E_{z}$ along the bunch, and has, so far, not been demonstrated with the desired controllability and beam quality.

As a novel approach to this problem, we illustrate our concept of a modulated plasma density in Fig. 1. The top panel shows the fields in the plasma cavity, Eqs. (1) and (2), for a density $n_{\min }=1 \times 10^{17} \mathrm{~cm}^{-3}$, where the bunch is at an accelerating and focusing phase. For a fixed delay $\zeta / c$, but higher density $n_{\max }=3 n_{\min }$, the bunch is defocused, but accelerated by a field of opposite slope and higher amplitude. Shifting the bunch between both phases with a phase $\Psi$

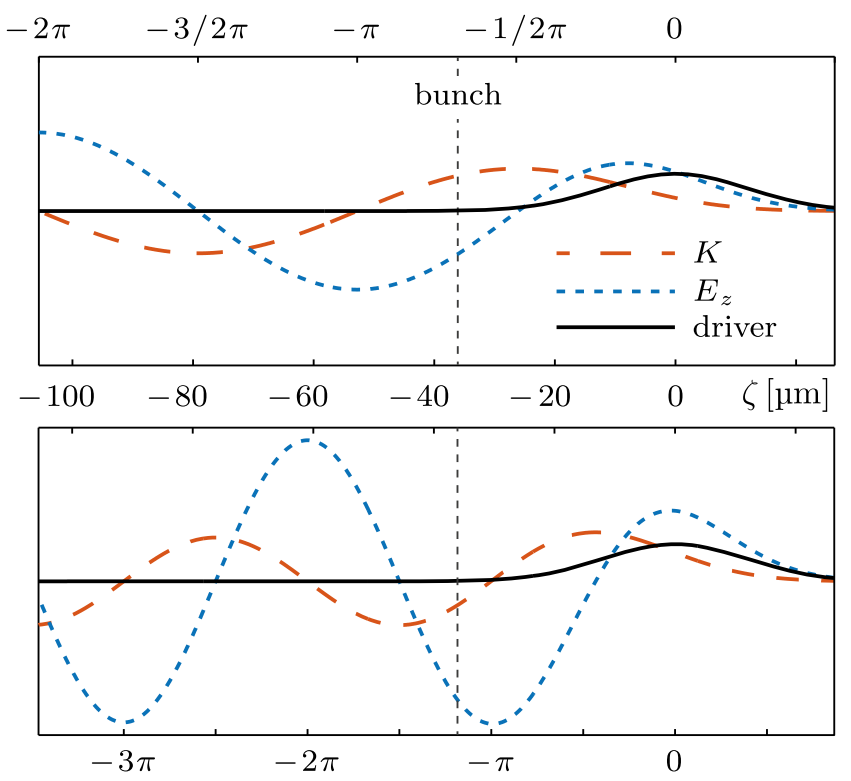

FIG. 1. On-axis accelerating field $E_{z}$ (arb. units) and focusing strength $K$ (arb. units) in a plasma cavity for a density $n_{\min }=$ $1 \times 10^{17} \mathrm{~cm}^{-3}$ (top) and $n_{\max }=3 n_{\min }$ (bottom). This corresponds to a plasma wavelength of 106 and $61 \mu \mathrm{m}$, respectively.

periodically modulated plasma density then creates on average a flat longitudinal field at the bunch position and thus mitigates the chirp evolution.

Successful implementation of the concept requires the beta function of the electron beam to be matched to the focusing of the modulated plasma density. In general, an electron under the influence of a constant focusing force $K$ is propagated along $z$ by the transport matrix $M$ :

$$
\left(\begin{array}{c}
r \\
r^{\prime}
\end{array}\right)=M\left(\begin{array}{c}
r_{0} \\
r_{0}^{\prime}
\end{array}\right), \quad \text { with } \quad M=\left(\begin{array}{c}
C S \\
C^{\prime} S^{\prime}
\end{array}\right)
$$

Here, $C=\cos (\sqrt{K} z)$ and $S=K^{-1 / 2} \sin (\sqrt{K} z)$, with $r$ the transverse coordinate of the electron, and prime $\left({ }^{\prime}\right)$ the derivative with respect to $z$. To describe the transport over one period $\lambda_{\text {mod }}$ of the modulated plasma density, we approximate the focusing profile $K=K(z)$ by a sequence of $i=1, \ldots, n$ stepwise constant $K_{i}$. The transport matrix over $\lambda_{\text {mod }}$ is then $T=M_{n} \times \cdots \times M_{1}$. Matched transport requires $|\operatorname{Tr}(T)|<2$ [16]. To describe the evolution of the whole electron beam, we use the Courant-Snyder parameters $\alpha=-\left\langle x x^{\prime}\right\rangle / \epsilon, \beta=\left\langle x^{2}\right\rangle / \epsilon$, and $\gamma_{T}=\left(1+\alpha^{2}\right) / \beta$, with $\epsilon$ the transverse emittance. They are related to the transport matrix

$$
T=\left(\begin{array}{c}
a b \\
c d
\end{array}\right)=\llbracket \cos (\mu)+J \sin (\mu), \text { with } J=\left(\begin{array}{c}
\alpha \beta, \\
-\gamma_{T}-\alpha
\end{array}\right),
$$

with $\llbracket$ being the identity matrix, $\mu$ the phase advance, $2 \cos (\mu)=\operatorname{Tr}(T)$, and $\sin (\mu)=\sqrt{1-\cos ^{2}(\mu)}$. From this, 
we can derive the beta function that is matched to a periodic structure, in our case the beginning of the modulated plasma:

$$
\beta(\zeta)=\frac{2 b}{\sqrt{4-(a+d)^{2}}} .
$$

Since $K$ depends on $\zeta$, the distance between bunch and driver, also the transport matrix $T$ and consequently $\beta$ are a function of $\zeta$. Real values of $\beta(\zeta)$ correspond to matched beam transport. The net accelerating field at $\zeta$ is $\bar{E}_{z}(\zeta)=\lambda_{\text {mod }}^{-1} \int_{0}^{\lambda_{\bmod }} E_{z}(r=0, \zeta, z) d z$. We choose the position $\zeta$ of the electron bunch behind the driver such that (i) we obtain matched transport using Eq. (3) and (ii) the bunch is accelerated on crest of the averaged accelerating field. There, at the minimum of $\bar{E}_{z}$, we maximize the energy gain and mitigate the chirp evolution.

To demonstrate our scheme, we assume in the following a driver laser pulse of Gaussian shape, with $a_{0}=0.8$, $\tau c=9.7 \mu \mathrm{m} \mathrm{rms}$, and a spot size of $w_{0}=2 \sigma_{r}=50 \mu \mathrm{m}$. We further assume a plasma density modulated between $n_{\text {max }}=3 \times 10^{17} \mathrm{~cm}^{-3}$ and $n_{\min }=1 \times 10^{17} \mathrm{~cm}^{-3}$, and a modulation profile,

$$
k_{p}(z)=k_{p, \text { min }}+\left(k_{p, \text { max }}-k_{p, \text { min }}\right)\left|\sin \left(\pi z / \lambda_{\text {mod }}\right)\right|^{b},
$$

with $k_{p} \propto \sqrt{n_{e}}$ and modulation period $\lambda_{\bmod }=1 \mathrm{~mm}$. The parameter $b$ describes the steepness and width of the density modulation, with $b=4$ in our example.

Our scheme does not require a specific functional dependence of the density modulation. While we also tested step functions and sinusoidal modulations, the profile specified in Eq. (4) is extracted from a fully 3D computational fluid dynamic simulation using OPENFOAM [17]. It is closest to the profile we would expect in an experiment using a capillary-based series of gas jets with $1 \mathrm{~mm}$ spacing and nozzle diameter of $300 \mu \mathrm{m}$ to produce the desired modulation on top of the density plateau. A discussion on robustness against density fluctuations and parametric scaling is given in the Supplemental Material [18].

Using this density profile, we calculate $\beta(\zeta)$ and $\bar{E}_{z}(\zeta)$, shown in Fig. 2(b), and compare it with a reference case of constant density $n_{e}=2 \times 10^{17} \mathrm{~cm}^{-3}$, Fig. 2(a). Both cases have a singularity in the beta function, which marks the transition to phases where the modulated plasma density supports a matched beta function. In the reference case [Fig. 2(a)], all delays supporting a matched beta function have a gradient in the accelerating field and would thus imprint a chirp on the accelerated bunch. Contrarily, for the modulated plasma [Fig. 2(b)], we find a delay, marked with a black dot, with a minimum in $\bar{E}_{z}$ and a matched beta function. This delay is equivalent to on-crest acceleration in a conventional accelerator, and no chirp is imprinted on the bunch.
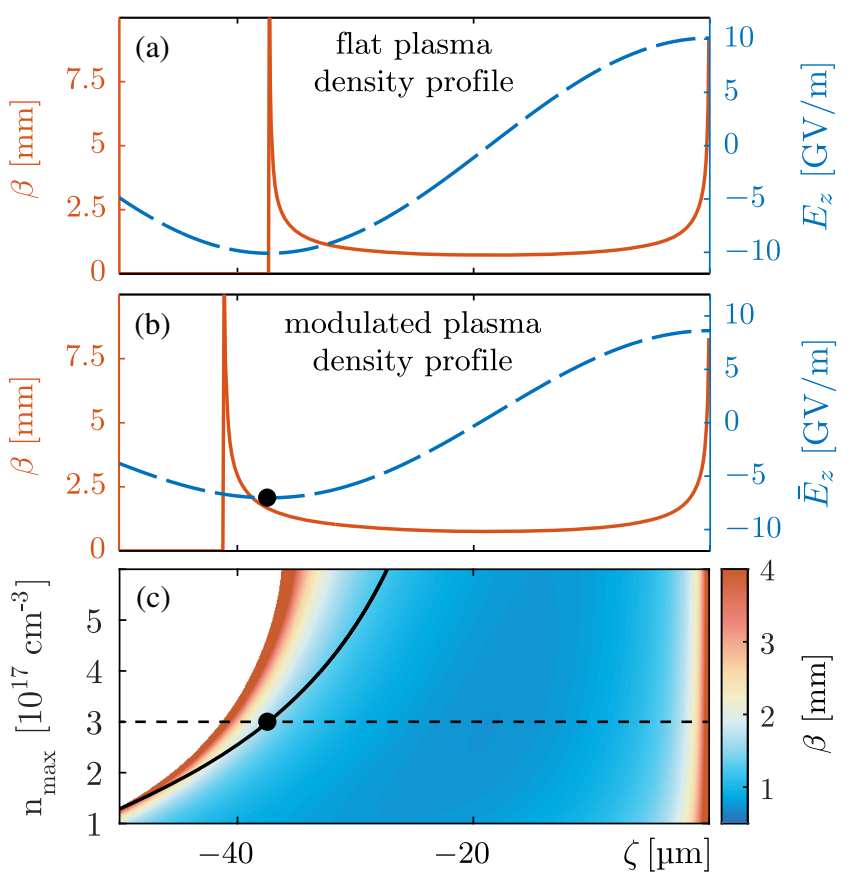

FIG. 2. Matched beta function (solid red line) and net accelerating field (dashed blue line), for a constant plasma density (a) and a modulated plasma (b), for which we find a distance $\zeta \approx-38 \mu \mathrm{m}$, equivalent to on-crest acceleration and matched transport. Panel (c) shows the color-coded matched beta function for different ratios of $n_{\max } / n_{\min }$. The position of minimum $\bar{E}_{z}$ (solid black) shifts towards the driver as $n_{\max }$ increases. Panel (b) is a lineout from (c) along the dashed line.

The formalism presented above can directly be applied to evaluate the influence of parameters like the density ratio $n_{\max } / n_{\min }$ or the modulation period $\lambda_{\text {mod }}$ on the created average fields. The density ratio $n_{\max } / n_{\min }$ can be used to control the position of minimum $\bar{E}_{z}$ within the region of stable transport, i.e., delays where a matched beta function exists. A larger ratio $n_{\max } / n_{\min }$ shifts the on-crest position closer to the driver and would also allow transport of longer bunches. This is illustrated in Fig. 2(c), where we show the color-coded matched beta function for different density ratios $n_{\max } / n_{\min }$. Here, we keep $n_{\min }=1 \times 10^{17} \mathrm{~cm}^{-3}$ fixed while we increase $n_{\max }$ to shift the minimum $\bar{E}_{z}$, which is marked by the black solid line, closer to the driver. Figure 2(b) is a lineout of Fig. 2(c) along the dashed line. The position of minimum $\bar{E}_{z}$ does not depend on the modulation period $\lambda_{\text {mod }}$. We find, however, that a threshold exists for $\lambda_{\text {mod }}$ where the beam can no longer be transported, as the defocusing region becomes too long. Lower peak plasma densities $n_{\max }$ allow longer $\lambda_{\text {mod }}$, while higher densities, due to the stronger defocusing fields, require shorter $\lambda_{\text {mod }}$. For our specific parameter set we find an upper limit $\lambda_{\text {mod }} \approx 3 \mathrm{~mm}$-much larger than the $1 \mathrm{~mm}$ period length we use in our example above.

To validate our results, we perform 3D PIC simulations using the code WARP [19] in the Lorentz boosted frame. 
The simulation box volume is $122 \times 200 \times 200 \mu \mathrm{m}^{3}$ with $4892 \times 100 \times 100$ cells and one particle per cell. The simulation is boosted by $\gamma_{\text {boost }}=8$. We inject an external electron beam of $100 \mathrm{MeV}$, with a bunch length of $\sigma_{z}=1 \mu \mathrm{m}$, and a normalized emittance of $\epsilon_{n}=$ $0.5 \mathrm{mmmrad}$. The transverse size of $\sigma_{x / y}=2 \mu \mathrm{m}$ is matched to the alternating focusing structure, using the simulated focusing forces and Eq. (3). The bunch has a relative energy spread of $\sigma_{\gamma} / \gamma=0.1 \%$, no longitudinal chirp, and a bunch charge of $1 \mathrm{pC}$ to avoid beam loading effects in this example. The distance between driver laser and witness bunch is chosen slightly larger than the analytical estimation with $\Delta \zeta=-40 \mu \mathrm{m}$ to account for the relativistically elongated plasma wavelength and slippage effects. For reference, we perform a simulation with the same parameters but with a constant flattop plasma profile of density $n_{e}=2 \times 10^{17} \mathrm{~cm}^{-3}$, and here, $\Delta \zeta=-39 \mu \mathrm{m}$.

Figure 3 shows the evolution of the correlated energy spread (chirp) for both cases. In the reference case (red dashed line) the bunch is in the focusing region, thus off crest of the accelerating field, and develops a steadily increasing energy chirp. In contrast, using a modulated plasma density, the energy chirp is compensated after every modulation period $\lambda_{\text {mod }}$. As the electron bunch is faster than the laser in the plasma, it slowly slips through the minimum in $\bar{E}_{z}(\zeta)$, compare Fig. 2(b), which causes the small global variation of the chirp. This effect could, however, be compensated with a globally tapered plasma density [20], and is not a limitation of our scheme. We did not

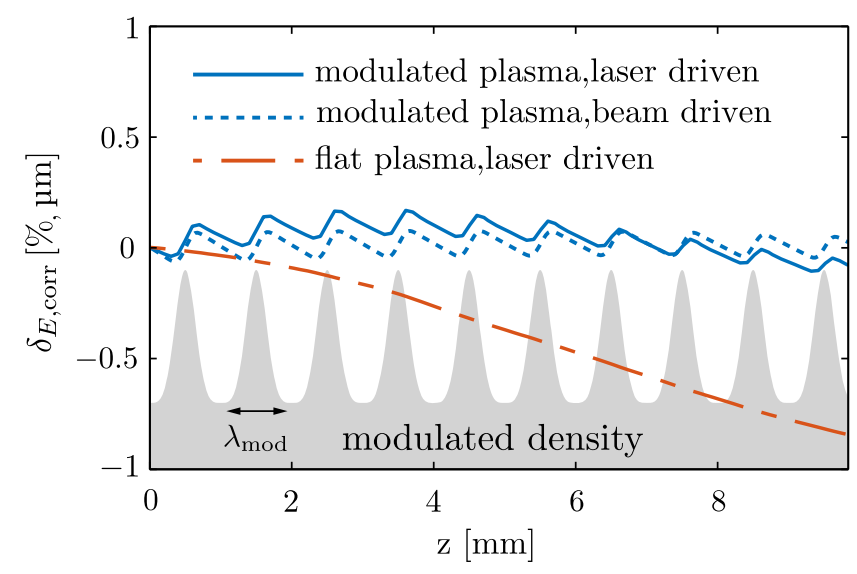

FIG. 3. Evolution of the correlated energy spread (chirp) $\delta_{E, \text { corr }}=\left\langle z E_{\text {kin }}\right\rangle /\left\langle z^{2}\right\rangle \bar{E}_{\text {kin }}$ from the laser- and beam-driven simulations. Compare main text for parameters. The chirp evolution for the constant focusing reference case is indicated in red (dash-dotted line), while the blue line (solid) shows the chirp evolution using the modulated plasma density. In contrast to the laser-driven case, there is no slippage between driver and witness electron bunch in an electron beam driven wakefield (blue dotted line), and we see no global variation in the correlated energy spread. The gray shaded area indicates the modulated plasma density profile. apply such a taper in our example to concentrate on the effect of the modulated plasma. Over one $\lambda_{\text {mod }}$ the chirp builds up faster than it decreases, which is a consequence of the difference in the slope of the accelerating field gradient depending on the plasma density. The beam emittance is conserved throughout the entire plasma.

We further investigate the modulated plasma density scheme for an electron beam-driven wakefield with 3D simulations using the PIC code HIPACE [21] with a simulation box volume of $118 \times 235 \times 235 \mu \mathrm{m}^{3}$ with $512 \times 320 \times 320$ cells. For this purpose, we assume a Gaussian driver with $\sigma_{z}=9.7 \mu \mathrm{m}, \sigma_{r}=25 \mu \mathrm{m}$, and $\epsilon_{n}=2.5 \mathrm{~mm} \mathrm{mrad}$. The driver peak density of $n_{b}=2.6 \times 10^{16} \mathrm{~cm}^{-3}$ corresponds to a charge of $400 \mathrm{pC}$. For simplicity, we choose in this conceptual study a driver beam energy of $20 \mathrm{GeV}$, to prevent an evolution of the driver, with an energy spread of $0.1 \%$. The witness bunch has identical parameters as in the laserdriven case.

We use the same modulated plasma profile as before and select the driver beam density such that the ratio $f_{0}=n_{b} / n_{e}\left(\bar{k}_{p}\right)=a_{0}^{2} / 4$ is the same as in the laser-driven case, which makes both comparable. Analogous to the laser driven case, we calculate $\beta(\zeta)$ and $\bar{E}_{z}(\zeta)$, and choose $\Delta \zeta=-43 \mu \mathrm{m}$ such that the witness is in the minimum of $\bar{E}_{z}$.

As expected, our simulations show that the energy chirp of the accelerated bunch is compensated, as in the laserdriven case; see Fig. 3. Since there is no slippage between witness and driver, we find no modulation in the global chirp evolution, and conclude that our scheme works for both laser- and beam-driven scenarios.

In Fig. 4, we compare the witness beam phase space of the laser-driven modulated plasma case to the reference case, both at the end of the plasma. Using the modulated plasma density, the bunch shows no residual linear energy

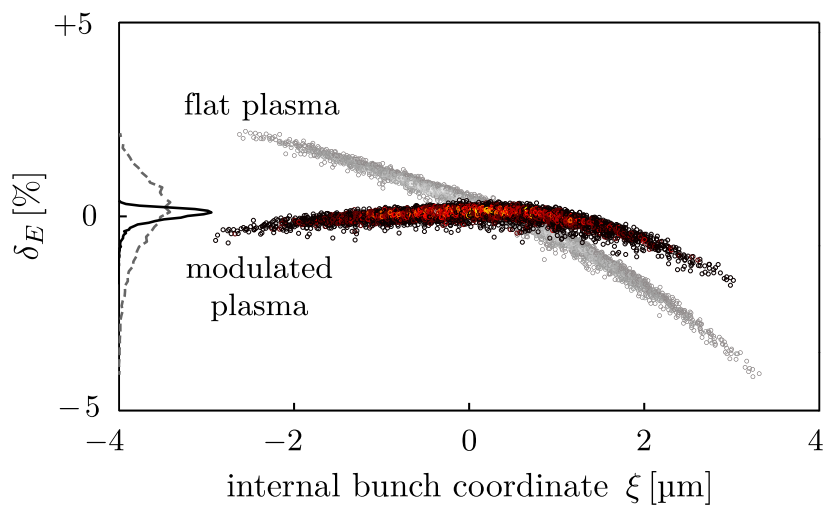

FIG. 4. Electron beam phase space after the propagation through the plasma. Here, $\delta_{E}=\Delta E_{\text {kin }} / \bar{E}_{\text {kin. }}$. In the reference case (gray) the beam has accumulated a negative chirp, while the chirp is compensated using a modulated plasma density. For the chosen parameter set, the mean beam energy is $\bar{E}_{\mathrm{kin}}=157 \mathrm{MeV}$ for the modulated plasma density and $\bar{E}_{\text {kin }}=181 \mathrm{MeV}$ for the reference case. 
chirp, unlike the reference case (gray). The projected rms energy spread is reduced by a factor of 4 to $0.24 \%$, compared to $0.96 \%$ in the reference case.

A unique and unprecedented feature of the modulated plasma density is the ability to actively shape the net acceleration field acting on the witness. Using macroscopic and experimentally accessible parameters, i.e., the plasma target geometry and gas flow into the target, the exact shape of the field is determined by the two densities $n_{\min }$ and $n_{\max }$. Choosing the densities such that the witness bunch is shifted between the accelerating and decelerating phases of the wakefield, the bunch experiences longitudinal fields of opposite slope and of opposite curvature. Thereby, we can, in principle, also generate a flat net accelerating field to remove the second order correlation from the bunch phase space.

As presented, our scheme is based on weakly beam loaded wakefields, expected, for example, in external injection schemes. It therefore shares the constraint with many plasma acceleration concepts of being well suited mainly for low charge bunches. For our specific parameter set, we see no quality degradation up to $4 \mathrm{pC}$ witness beam charge. For $10 \mathrm{pC}$, corresponding to a ratio of beam density to plasma density of $\sim 5$, we find a deterioration of beam emittance and projected energy spread. Solutions exist to accelerate higher charges while limiting the effect of the witness-generated wakefield, and include larger driver spot sizes or transversely shaped driver profiles [22].

In conclusion, we propose a novel scheme based on a modulated plasma density to actively shape the net accelerating field acting on the witness bunch in a plasma wakefield. Our concept effectively suppresses the buildup of large energy chirps during acceleration, while conserving the beam emittance, which is crucial for any staged acceleration scheme. It thus promises to overcome one of the major challenges in the field, the generation of highquality electron beams.

We gratefully acknowledge the computing time provided on the supercomputer JURECA under project HHH20. We acknowledge the use of the High-Performance Cluster (IT-HPC) at DESY. This work was funded in part by the Humboldt Professorship of B. Foster, the DAAD, and the Helmholtz Virtual Institute VH-VI-503.

*andreas.maier@desy.de

[1] V. I. Veksler, Sov. J. At. Energy 2, 525 (1957).
[2] E. Esarey, C. B. Schroeder, and W. P. Leemans, Rev. Mod. Phys. 81, 1229 (2009).

[3] C. Rechatin, J. Faure, A. Ben-Ismail, J. Lim, R. Fitour, A. Specka, H. Videau, A. Tafzi, F. Burgy, and V. Malka, Phys. Rev. Lett. 102, 164801 (2009).

[4] A. R. Maier, A. Meseck, S. Reiche, C. B. Schroeder, T. Seggebrock, and F. Grüner, Phys. Rev. X 2, 031019 (2012).

[5] Z. Huang, Y. Ding, and C. B. Schroeder, Phys. Rev. Lett. 109, 204801 (2012).

[6] C. Schroeder, E. Esarey, C. Geddes, C. Benedetti, and W. Leemans, Phys. Rev. ST Accel. Beams 13, 101301 (2010).

[7] G. Fubiani, E. Esarey, C. B. Schroeder, and W. P. Leemans, Phys. Rev. E 70, 016402 (2004).

[8] S. Steinke, J. van Tilborg, C. Benedetti, C. G. R. Geddes, C. B. Schroeder, J. Daniels, K. K. Swanson, A. J. Gonsalves, K. Nakamura, N. H. Matlis, B. H. Shaw, E. Esarey, and W. P. Leemans, Nature (London) 530, 190 (2016).

[9] R. Assmann and K. Yokoya, Nucl. Instrum. Methods Phys. Res., Sect. A 410, 544 (1998).

[10] T. Mehrling, J. Grebenyuk, F. S. Tsung, K. Floettmann, and J. Osterhoff, Phys. Rev. ST Accel. Beams 15, 111303 (2012).

[11] I. Dornmair, K. Floettmann, and A. R. Maier, Phys. Rev. ST Accel. Beams 18, 041302 (2015).

[12] X. L. Xu, J. F. Hua, Y. P. Wu, C. J. Zhang, F. Li, Y. Wan, C.-H. Pai, W. Lu, W. An, P. Yu, M. J. Hogan, C. Joshi, and W. B. Mori, Phys. Rev. Lett. 116, 124801 (2016).

[13] L. M. Gorbunov and V. I. Kirsanov, Sov. Phys. JETP 66, 290 (1987).

[14] T. C. Chiou and T. Katsouleas, Phys. Rev. Lett. 81, 3411 (1998).

[15] T. C. Katsouleas, S. Wilks, P. Chen, J. M. Dawson, and J. J. Su, Part. Accel. 22, 81 (1987).

[16] E. D. Courant and H. S. Snyder, Ann. Phys. (Paris) 3, 1 (1958).

[17] H. G. Weller, G. Tabor, H. Jasak, and C. Fureby, Comput. Phys. 12, 620 (1998).

[18] See Supplemental Material at http://link.aps.org/ supplemental/10.1103/PhysRevLett.118.214801 for a discussion on robustness against density fluctuations and parametric scaling.

[19] A. Friedman, R. H. Cohen, D. P. Grote, S. M. Lund, W. M. Sharp, J.-L. Vay, I. Haber, and R. A. Kishek, IEEE Trans. Plasma Sci. 42, 1321 (2014).

[20] W. Rittershofer, C. B. Schroeder, E. Esarey, F. J. Grner, and W. P. Leemans, Phys. Plasmas 17, 063104 (2010).

[21] T. Mehrling, C. Benedetti, C. B. Schroeder, and J. Osterhoff, Plasma Phys. Controlled Fusion 56, 084012 (2014).

[22] E. Cormier-Michel, E. Esarey, C. G. R. Geddes, C. B. Schroeder, K. Paul, P. J. Mullowney, J. R. Cary, and W. P. Leemans, Phys. Rev. ST Accel. Beams 14, 031303 (2011). 